Merge of motion analysis, multibody dynamics and finite element method for the subject-specific analysis of cartilage loading patterns during gait : differences between rotation and moment-driven models of human knee joint

\title{
Klodowski, Adam
}

2016-07

Klodowski , A, Mononen , M E , Kulmala , J P , Valkeapaa , A, Korhonen, R K, Avela , J , Kiviranta , I , Jurvelin , J S \& Mikkola , A 2016 , ' Merge of motion analysis, multibody dynamics and finite element method for the subject-specific analysis of cartilage loading patterns during gait : differences between rotation and moment-driven models of human knee joint ' , Multibody System Dynamics , vol. 37 , no. 3 , pp. 271-290 . https://doi.org/10.1007/s11044-015-9470-y

http://hdl.handle.net/10138/224051

https://doi.org/10.1007/s11044-015-9470-y

publishedVersion

Downloaded from Helda, University of Helsinki institutional repository.

This is an electronic reprint of the original article.

This reprint may differ from the original in pagination and typographic detail.

Please cite the original version. 


\title{
Merge of motion analysis, multibody dynamics and finite element method for the subject-specific analysis of cartilage loading patterns during gait: differences between rotation and moment-driven models of human knee joint
}

\author{
Adam Kłodowski ${ }^{1}$ - Mika E. Mononen ${ }^{2}$ Juha P. Kulmala ${ }^{3}$ Antti Valkeapää ${ }^{1}$. \\ Rami K. Korhonen ${ }^{2}$ Janne Avela ${ }^{3}$. Ilkka Kiviranta ${ }^{4} \cdot$ Jukka S. Jurvelin $^{2}$. \\ Aki Mikkola ${ }^{1}$ \\ Received: 24 July 2014 / Accepted: 13 July 2015 / Published online: 29 July 2015 \\ (C) Springer Science+Business Media Dordrecht 2015
}

\begin{abstract}
Understanding joint loading is important when evaluating sports training methods, sports equipment design, preventive training regimens, post-op recovery procedures, or in osteoarthritis' etiology research. A number of methods have been introduced to estimate joint loads but they have been limited by the lack of accuracy in the joint models, including primarily the lack of patient-specific motion inputs in the models with sophisticated, fibril-reinforced material models. The method reported here records and applies patient-specific human motion for in-depth cartilage stress estimation. First, the motion
\end{abstract}

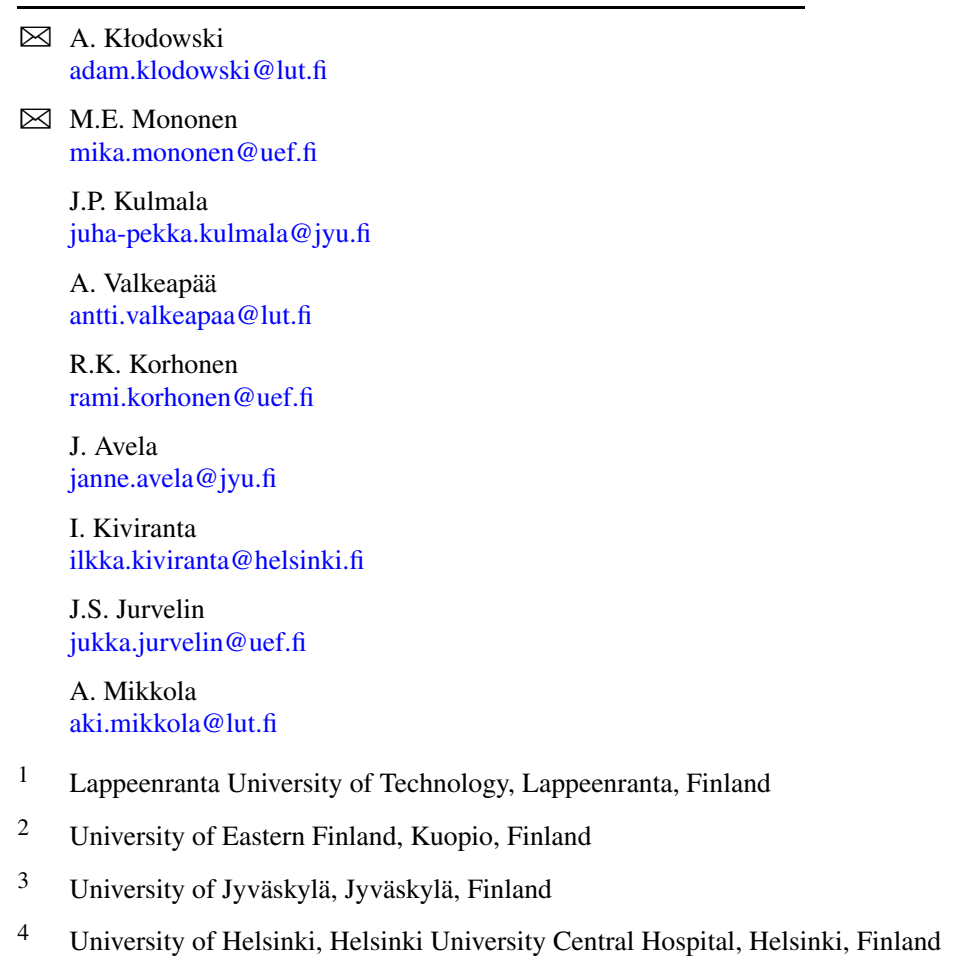


analysis of a subject was conducted. Due to skin motion, multibody simulation was used to correct motion capture. These data was used as an input in a finite element model. The model geometry was based on magnetic resonance imaging and cartilage was modeled as a fibril-reinforced poroviscoelastic material. Based on the experimental motion data (motion analysis and multibody simulation), two models were created: a rotation-controlled and a moment-controlled model. For comparison, a model with motion input from the literature was created. The rotation-controlled model showed the most even stress distribution between lateral and medial compartments and smallest stresses and strains in a depth-wise manner. The model based on the literature motion simulated very high stresses and uneven stress distribution between the joint compartments. Our new approach to determine dynamic knee cartilage loading enables estimations of stresses and strains for a specific subject over the entire motion cycle.

Keywords Menisci · Fibril-reinforced · Kinesiology · Computational model · Cartilage · Fibril-reinforced $\cdot$ Poroelastic

\section{Introduction}

Osteoarthritis (OA) is a common musculoskeletal disease that leads to disability and considerable cost to society $[1,2]$. Although the exact mechanisms of knee OA are unclear, excessive loading is thought to be a major contributing factor for the onset and progression of post-traumatic OA [3, 4]. Anterior cruciate ligament (ACL) and meniscal injuries can lead to abnormal knee joint mechanics [5]. However, little is understood about how joint loads are distributed among ligaments, periarticular muscles, menisci, and cartilage [6] and about the role of the neuromuscular system in knee OA [7]; we still do not fully understand the mechanisms for its pathogenesis. No clinically feasible measurement methods exist to accurately determine in-vivo intact knee motion, joint loading, and tissue deformation that occur during typical physical activities. Therefore, measurement techniques, in conjunction with computational methods (e.g., multibody formalisms and finite element (FE) method), should be used to study mechanical factors that contribute to the risk of knee OA. Consequently, this information may then be used to retard or even prevent its progression.

The human musculoskeletal system can be idealized as a multibody system where bone segments are interconnected via joints, and muscles are considered as force actuators. Multibody musculoskeletal analysis can be divided into inverse [8,9], and forward dynamics $[10,11]$. Inverse dynamics is a kinematics-based procedure that enables the solution of unknown joint torques to estimate muscular forces [12]. Forward dynamics, in contrast, utilizes forces to determine model kinematics. These forces can be determined using contraction or joint rotation patterns computed via inverse dynamics [13]. Furthermore, we can factor bone flexibility into the analysis $[14,15]$. In turn, the role of bone geometry and subchondral bone changes on OA development can be studied [16].

The nonlinear material behavior of cartilage under loading has been well recognized $[17,18]$. Using multibody assessment, clinically valuable results could be obtained for cartilage mechanics in a non-invasive manner and reasonable timeframe. To assess the stressstrain behavior of cartilage, tissue level FE models are needed.

Cartilage strains and stresses and the stress absorption of menisci in human knee joints have been studied previously using the FE method [19-26]. FE models can capture the geometry of articular surfaces in the knee, the mechanical nonlinearity of the joint tissues, 
and interactions between the solid and fluid phases in the cartilage. However, due to complex tissue structure and properties, cartilage material behavior can rarely be described realistically [24-26]. The main structural components of the articular cartilage solid matrix and fluid determine the time-dependent response of this tissue during joint loading. The realistic tissue structure has been taken into account in fibril-reinforced biphasic models of cartilage [27, 28]. Recently, a detailed fibril-reinforced biphasic cartilage material with depth-dependent tissue properties was implemented in knee models [19-23]. However, the information on joint loading in these models is highly simplified and/or taken from the literature. As joint movement differs from subject-to-subject [29], it is advantageous to include subject-specific joint loads, preferably during physiological activity, as an input for the knee joint models.

We establish a two-level modeling approach that combines subject-specific motioncaptured gait data, magnetic resonance imaging (MRI) of the knee joint, multibody simulation, and a fibril-reinforced biphasic FE model of articular cartilage to predict the tibiofemoral contact parameters and depth-wise cartilage stresses and strains. Differences in these parameters are emphasized, especially between the rotation and moment-driven knee joint models. Further, we compare the results obtained from this subject-specific approach to the predictions of the reference model where motion data is obtained from the literature.

\section{Materials and methods}

We established a stepwise procedure to predict the knee cartilage loading of a walking human subject (Figs. 1 and 2). First, motion was captured and ground reaction force (GRF) and anthropometric measurements were taken, followed by an MRI scanning procedure. The sagittal-plane range of motion observed during motion capture was used as an input into the intermediate FE model, which made it possible to determine the relationship between varus-valgus and sagittal-plane rotation. The data was then implemented into the multibody simulation, which computed muscle forces and all three knee rotation angles. The multibody

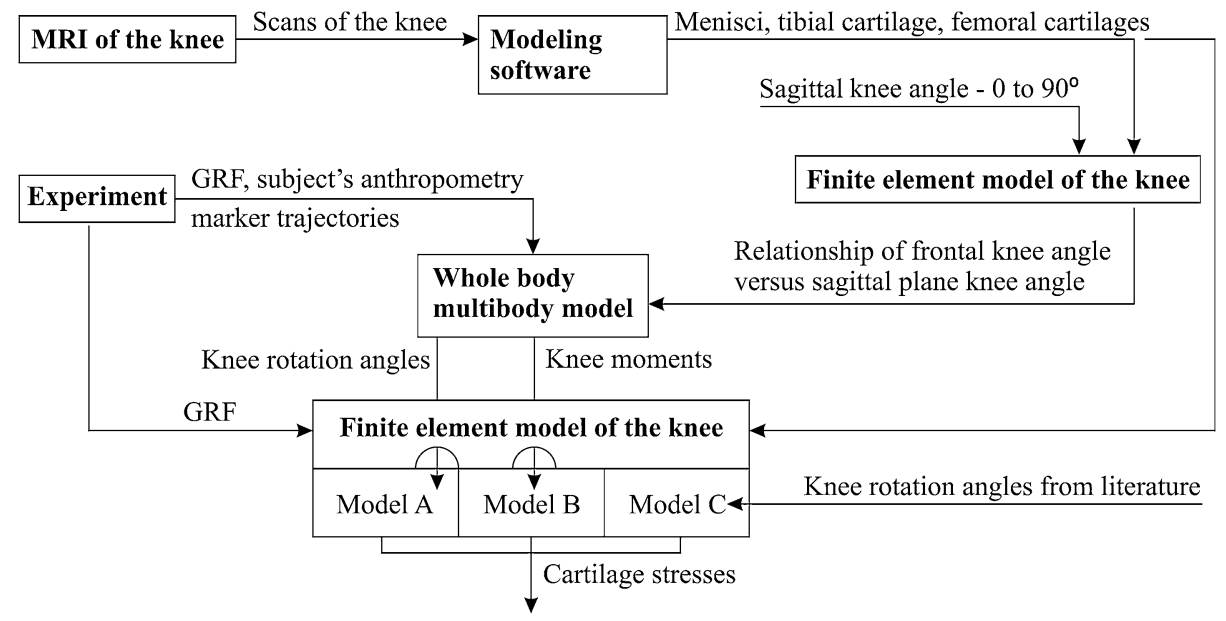

Fig. 1 Workflow of the study 


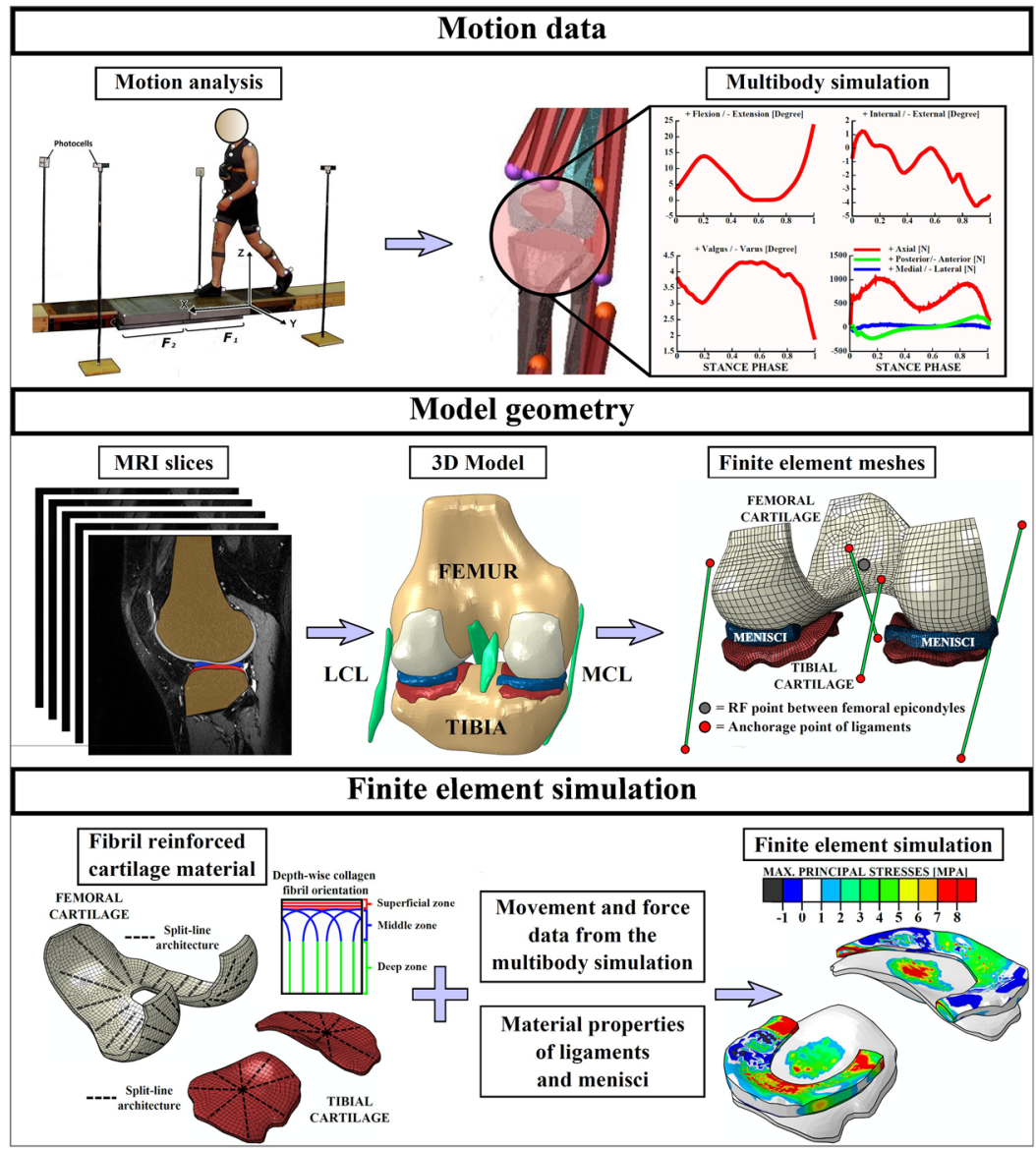

Fig. 2 Workflow of the study, including steps from motion analysis through multibody simulation (first row) to knee joint FE model (middle and bottom rows), showing the generation of the FE mesh from MRI (middle row) and implementation of material properties and patient specific motion (bottom row, Fig. 5)

results provided the input for the detailed FE model, which enabled the prediction of load distribution within the knee joint cartilage during walking. Knee model geometry was reconstructed from the MRI data of the knee joint. Alignment of the knee models in multibody and finite element software was achieved by using the same local coordinate system. As the motion data transferred between multibody software and finite element tool was in the form of knee rotations, there was no need to transfer motion capture markers between multibody and FE models. However, motion capture markers were synchronized with the position of the markers on the multibody model using the least squares fit. In addition, the same global coordinate system was used during the experiment and in the multibody model.

The paper presents three detailed knee models, named Model A, B, and C, respectively (see Fig. 5). All models share the same finite element representation, but differ in actuation method. Model A is driven by imposed rotations and forces obtained from the multibody model, Model B uses moments and forces as the actuation source, while Model C uses rotation, translation and forces data from literature (not subject-specific) for reference. 


\subsection{Data collection and the measurement setup}

Prior to motion capture, static anthropometric measurements of a healthy human subject (height, weight, leg length, and knee and ankle diameters) were conducted. Next, thirty-four $14 \mathrm{~mm}$ retro-reflective markers were attached to the subject according to the Plug-in Gait full body model (Vicon Motion Systems). Our earlier experience showed that this number of markers is not sufficient to capture subtle varus-valgus and internal-external rotations of the knee joint simply due to skin motion artifact. Therefore, eight additional markers were placed on the anterior side of the thigh (four markers), the anterior side of the tibia (two markers), the medial knee joint line (one marker), and the patella (one marker).

With the subject walking at his normal speed of $1.7 \mathrm{~m} / \mathrm{s}$, a ten-camera Vicon system (T40) and a force platform (AMTI OR6-6, Advanced Mechanical Technology, Inc. Watertown, MA, USA) were used to record marker positions and reaction forces synchronously at 500 and $1500 \mathrm{~Hz}$, respectively (Fig. 1, top-left). The marker trajectories and GRF data were low-pass filtered using a zero-lag fourth order Butterworth filter with cut-off frequencies of 6 and $20 \mathrm{~Hz}$, respectively. In addition to detailed multibody inverse dynamics analysis, simple inverse dynamics simulation provided by Vicon software (Nexus v1.7) was used to calculate knee rotation moments (internal-external and varus-valgus) for reference.

\subsection{Magnetic resonance imaging}

To obtain a detailed, subject-specific joint geometry, the left knee of a clinically healthy 28 -year old Caucasian male patient $(1.77 \mathrm{~m}, 82 \mathrm{~kg})$ was imaged using a standard Achieva 3.0T MRI system (Philips Healthcare, Best, The Netherlands). Imaging was conducted using a 3D, isotropic Proton Density Weighted (PDW) Fast Spin Echo (FSE) sequence, in other words, the Phillips Medical Systems' volumetric isotropic turbo spin-echo acquisition (VISTA) sequence. The parameters used were TR $=1300 \mathrm{~ms}, \mathrm{TE}=32.3 \mathrm{~ms}$, in-plane resolution $0.5 \mathrm{~mm}$, and slice thickness $0.5 \mathrm{~mm}$ (Fig. 2, middle-left). The knee joint was imaged in flexion and at valgus angles of $9^{\circ}$ and $3^{\circ}$, respectively. Imaging was conducted according to the ethical guidelines of Kuopio University Hospital, Finland. MR imaging was conducted with the permission (94/2011) from the local ethical committee of the Kuopio University Hospital, Kuopio, Finland, and written consent was obtained from the volunteer.

\subsection{Multibody Model}

Recording human movement to estimate knee joint motion is complicated by the relative movement between the skin, and the internal knee structure, which was observed during the experiment. Because of the possible skin motion errors, the acquired motion system data was deemed insufficient to reliably define the internal-external or varus-valgus rotations within the knee joint. Therefore, a full body multibody model was created. The human musculoskeletal multibody model was constructed using the LifeMOD virtual human modeling plug-in and general purpose MD ADAMS simulation software (version 2010.00.3388, MSC Software Corporation). The skeletal model was based on the data in the statistical anthropometric measurements database [30,31]. The computer model was scaled using the subject's weight, height, gender, and age as parameters. The skeleton consisted of 17 bodies connected by 16 spherical (3 rotational degrees of freedom) kinematic joints (Fig. 2, top-right). Muscle model representations were added as appropriate to complete the musculoskeletal model. The joints and muscles were modeled as in the previous publication [10], with the exception for the knee joint, where translational compliance was introduced in the 
Fig. 3 Foot contact element placement and geometry $\underline{\text { Isometric view }}$

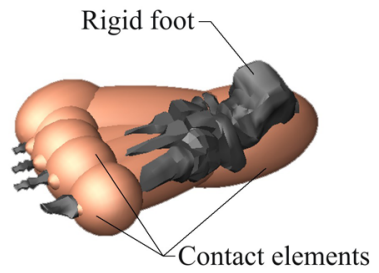

Botom view

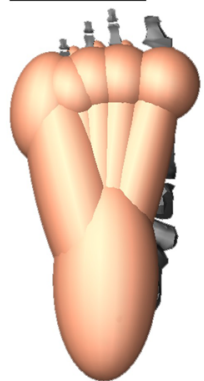

current model using bushing elements and all three knee rotations were allowed. ADAMS software's bushing element represents three orthogonal springs between two bodies allowing relative motion between the bodies constrained by spring forces. Finally, the model had 57 degrees of freedom and was actuated by 118 muscles. A proportional-integral-derivative controller that uses desired and current muscle length as input signals was used to control muscle contraction. The physiological limits of muscle force production were implemented using conditional expressions in the muscle controller. The muscle force limits were based on the statistical database available in LifeMod software and scaled to match subject's size. LifeMod muscle parameter database represents data defined by Eycleshymer and Schoemaker [32]. The optimization of the muscle forces with minimum total force production as a target function was used. All bones were modeled as rigid bodies with inertia properties, based on statistics, corresponding to subject anthropometry.

Multi-element contact model was prepared for each foot. The contact model consists of two steps. The first is the geometric contact detection procedure, and the second is the calculation routine for contact force. In this study both routines use the same contact geometry. The contact geometry of the foot is simplified to ellipsoids; the ground geometry is represented by a single plane. The ellipsoid-plane contact model runs efficiently and results in quick contact calculations. Normal component, $F_{N}$, of the contact force $F$ is calculated based on penetration depth $s$ using the following formula:

$$
F_{N}=K s^{n}+c(s) \dot{s},
$$

where $K$ is the contact stiffness, $n$ is the exponent coefficient, $\dot{s}$ is the velocity of penetration, and $c(s)$ is the damping coefficient function dependent on penetration value. Penetration depth is defined as the shortest distance between the centroid of the contact volume of the ellipsoid that penetrates through the contact plane. The normal component of the contact force is perpendicular to the ground contact plane. It is represented at the center of the volume by three force components and three moments. Coulomb's friction model is used with velocity dependent friction coefficient to model friction between feet and ground. The ground was modeled as a rigid plane and on each foot, 11 ellipsoid contact elements were placed as shown in Fig. 3. More details on the contact model used are presented in [33].

Motion-capture data provided the primary input needed to perform an inverse dynamics simulation. During inverse dynamic simulation, translational compliance in the knee, contact model between ground and feet, and muscle actuators are disabled. During the inverse dynamics simulation, muscle contraction patterns are recorded as reference data for muscle controllers utilized in forward dynamics. After inverse dynamics simulation is complete, muscle actuators, contact models, and translational compliance in the knee are enabled. The varus-valgus rotation measurement data at the knee joint was not accurate enough for the 


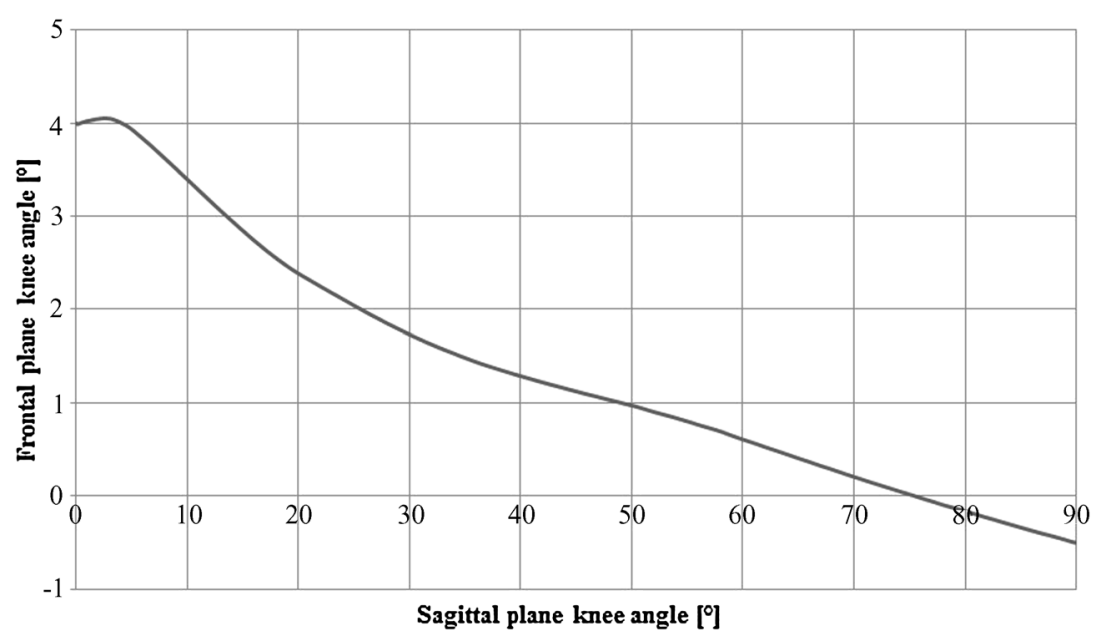

Fig. 4 Relationship between frontal and sagittal plane knee angle using intermediate finite element model

purpose of this study. Therefore, the knee joint was considered as a spherical joint with translational compliance in the multibody model, and a parametric constraint function was included to link the flexion-extension knee rotation with the varus-valgus rotation of the knee during gait. The relationship between flexion-extension and varus-valgus rotations was determined using a nonlinear FE analysis (see Sect. 2.2) because the geometry of the joint surfaces affects joint kinetics. This intermediate step allowed describing patientspecific variation in joint geometry in the multibody model. In this intermediate FE analysis the varus-valgus rotation was determined for the whole range of flexion-extension movement. Based on the simulation, the constraint function for the varus-valgus rotation was calculated as a function of the flexion-extension angle, which is presented in Fig. 4. In the multibody model, the function was implemented as algebraic constraint linking the input spline data and the simulation knee rotation parameter.

Muscle contraction trajectories obtained in the inverse dynamics simulation were used as an input to forward dynamics muscle controllers. A forward dynamics simulation was carried out, including the skeletal structures actuated by muscles. This simulation estimated the two unknown knee joint rotations (internal-external and varus-valgus). The geometric constraints imposed by knee cartilage, human body dynamics, and the contact model between the subject's feet and the ground were included in the simulation. Since there may occur small variation between individual gait cycles, two consecutive simulation cycles, with a time variance of $\leq 0.1 \mathrm{~s}$, were averaged to compose the motion data used later as the input for a detailed FE model of the knee (Fig. 2, top-right). Based on these data, the ground reaction force measurements, and the patient-specific knee geometry with ligaments, the FE analysis was used to determine the load distribution in the articular cartilage.

\subsection{FE-analysis}

\subsubsection{Model geometry and mesh}

The manual segmentation of knee joint tissues came from MRI slices using Mimics v12.3 (Materialise, Leuven, Belgium) medical image processing software. The femoral and tibial 
cartilage, the menisci, and the cruciate and collateral ligaments were constructed as a surface mesh (Fig. 2, middle).

Then, using a custom-made Matlab script (MathWorks, Natick, MA, USA), the surface mesh was converted into a solid format. Finally, the 3D solid parts of femoral and tibial cartilage and menisci (Fig. 2, middle-center) were imported into the FE software (Abaqus v6.1, Dassault Systèmes, Providence, RI, USA). To reduce computation times, bones were not included in the FE analysis. The model was meshed using linear 8-node hexahedral poroelastic elements (C3D8P) for cartilage and corresponding elastic elements (C3D8) for menisci (Fig. 2, middle-right). The characteristic element lengths of femoral and tibial cartilage and menisci were $1.45,0.61$, and $0.56 \mathrm{~mm}$, respectively, resulting in 3 and 6 depth-wise element layers to the femoral and tibial cartilage (Fig. 2, middle-right). In order to simplify the model and reduce computation time, ligaments were modeled as linear spring elements [34], and the bone-cartilage interfaces were assumed rigid. Before the final analysis, a mesh convergence test was conducted by comparing the results obtained from the aforementioned FE mesh to a model, in which a characteristic element length of tibial cartilage was $0.31 \mathrm{~mm}$. At the first peak loading force, the differences between the average pore pressures in the medial tibial cartilage at the tibio-femoral contact area were less than $1 \%$.

\subsubsection{Material properties of cartilage, meniscus and ligaments}

Similarly to previous studies $[18,28,35,36]$, femoral cartilage and tibial cartilage were modeled with fibril reinforced poroviscoelastic (FRPVE) material behavior using UMAT, a user-defined subroutine, in conjunction with Abaqus (Fig. 2, bottom). In the FRPVE material, the total stress $\left(\sigma_{t}\right)$ is the sum of the effective solid stress of the non-fibrillar matrix $\left(\sigma_{\mathrm{nf}}\right)$, the fibril network stress $\left(\sigma_{\mathrm{f}}\right)$, and the fluid pressure $\mathrm{p}$ as follows:

$$
\sigma_{\mathrm{t}}=\boldsymbol{\sigma}_{\mathrm{nf}}+\boldsymbol{\sigma}_{\mathrm{f}} \pm p \mathbf{I}
$$

where $\mathbf{I}$ is the unit tensor. The non-fibrillar matrix was modeled as a Neo-Hookean porohyperelastic material with the Young's modulus $\left(\mathrm{E}_{\mathrm{m}}\right)$ of $0.31 \mathrm{MPa}$, Poisson's ratio $\left(\mathrm{v}_{\mathrm{m}}\right)$ of 0.42 , and permeability $(\mathrm{k})$ of $1.74 \times 10^{-15} \mathrm{~m}^{4} / \mathrm{Ns}$ [36-38]. The fluid fraction was adjusted to vary linearly from the cartilage surface $(90 \%)$ to the cartilage-bone interface $(75 \%)$ [39, 40].

The fibrillar network was modeled to be viscoelastic with the initial and strain-dependent fibril network moduli $\left(\mathrm{E}_{\mathrm{f}}^{0}\right.$ and $\left.\mathrm{E}_{\mathrm{f}}^{\varepsilon}\right)$ of 0.47 and $673 \mathrm{MPa}$, and a damping coefficient $(\eta)$ of $947 \mathrm{MPa}$ s [28]. Similarly to a previous study [41], the collagen fibrils with a depthdependent arcade-like fibril architecture and split-line patterns were implemented into the model (Fig. 2, bottom) [42-46].

The meniscus was modeled as a linearly elastic transversely isotropic material without fluid [47-49]. The Young's modulus in the axial and radial directions was $20 \mathrm{MPa}$. The Young's modulus of $140 \mathrm{MPa}$ was used in the circumferential direction. The Poisson's ratios in-plane and out-of-plane were 0.2 and 0.3 , respectively. The shear modulus was $50 \mathrm{MPa}$ [47-49]. Elastic material parameters were considered acceptable for the meniscus because fluid flow is virtually negligible in dynamically compressed poroelastic materials (especially in case of low impact exercise as walking); furthermore, we were not interested in the fluid pressure of the meniscus. This also reduced computation times. Further, validated FRPVE material parameters for the meniscus are lacking. The meniscus was attached to the tibia using linear spring elements (number of spring elements $=$ number of nodes at the horn ends). The number of spring elements was 133 and 119 for the anterior and posterior horns of the medial meniscus, respectively, and 147 for the anterior and posterior horns of the 
lateral meniscus. At each horn, the total spring stiffness was adjusted to match $350 \mathrm{~N} / \mathrm{mm}$ [50].

Anterior cruciate ligament (ACL), posterior cruciate ligament (PCL), medial collateral ligament (MCL), and lateral collateral ligament (LCL) were modeled as linear spring elements (one spring per ligament). A stiffness of $200 \mathrm{~N} / \mathrm{mm}$ was implemented for ACL and PCL, and $100 \mathrm{~N} / \mathrm{mm}$ for MCL and LCL [34]. The optimal ligament pre-tension was sought to realistically mimic joint forces of unloaded knee and joint mechanics during walking. Several ligament pre-tensions (accuracy: $\pm 0.5 \mathrm{~mm}$ ) were tested, and pre-elongations of $1.5 \mathrm{~mm}$ produced consistent reaction forces with the experimentally measured forces (initial and peak loading forces) produced by [51].

\subsubsection{Implementation of motion, boundary conditions and simulations}

Gait cycle implementation was based either on joint angular changes derived from relative femoral-to-tibial rotation (Fig. 5, Model A), or moments (Fig. 5, Model B), simulated by the multibody model. In addition, in the last model gait input was taken from the literature $[23,29,52]$ for comparison (Fig. 5, Model C). For all the models, knee joint forces were the result of ligament pre-tension and measured GRF data (Fig. 5, top \& middle, right column). Identical extension-flexion rotations were used for models A and B (Fig. 5, top \& middle, left column).

The experimental motion and force curves (Fig. 5) were incorporated into a reference point (RP) of the FE model of the knee, as described in previous studies [22, 23], while the bone-tibial cartilage interface was fixed in all directions. The RP was defined and positioned at the middle between the lateral and medial epicondyles of the femur (Fig. 2, middle-right). The upper femoral cartilage surface (cartilage-bone interface) was attached to the RP using kinematic coupling constraint equations and the amplitude options offered by Abaqus. Frictionless surface-to-surface contacts with finite sliding were implemented for cartilage-to-cartilage and cartilage-to-meniscus contacts. This contact choice prevented free fluid flow through cartilage surfaces. Walking simulations for all three aforementioned models were conducted using soils consolidation analysis with nonlinear geometry. Knee rotations and translations were compared between the models. Reaction forces, contact pressures, maximum principal stresses and strains were analyzed from the tibio-femoral contact area. Depth-wise (from the cartilage surface to the bone) maximum principal stresses, fluid pressures and fibril strains were also analyzed for all models, and ligament elongations were computed for additional verification.

\section{Results}

The rotation-controlled model (Model A) gave the most even distribution of reaction forces and stresses between the lateral and medial tibial compartments; 32-80\% and 20-68\% of the total force, respectively (Fig. 6, left). The highest reaction forces occurred at $30 \%$ and $95 \%$ of the stance for the lateral tibial cartilage and $10 \%$ and $75 \%$ of the stance for the medial tibial cartilage (Fig. 6, left). The moment-controlled model (Model B) showed substantially higher joint forces in the medial compartment (26-100\%) than in the lateral 0$74 \%$ ) compartment (Fig. 6, middle). The maximum values of the reaction force in the medial compartment were found to coincide with the first and second peak loads. The reference model produced over $80 \%$ of the total reaction force in the medial compartment during the 


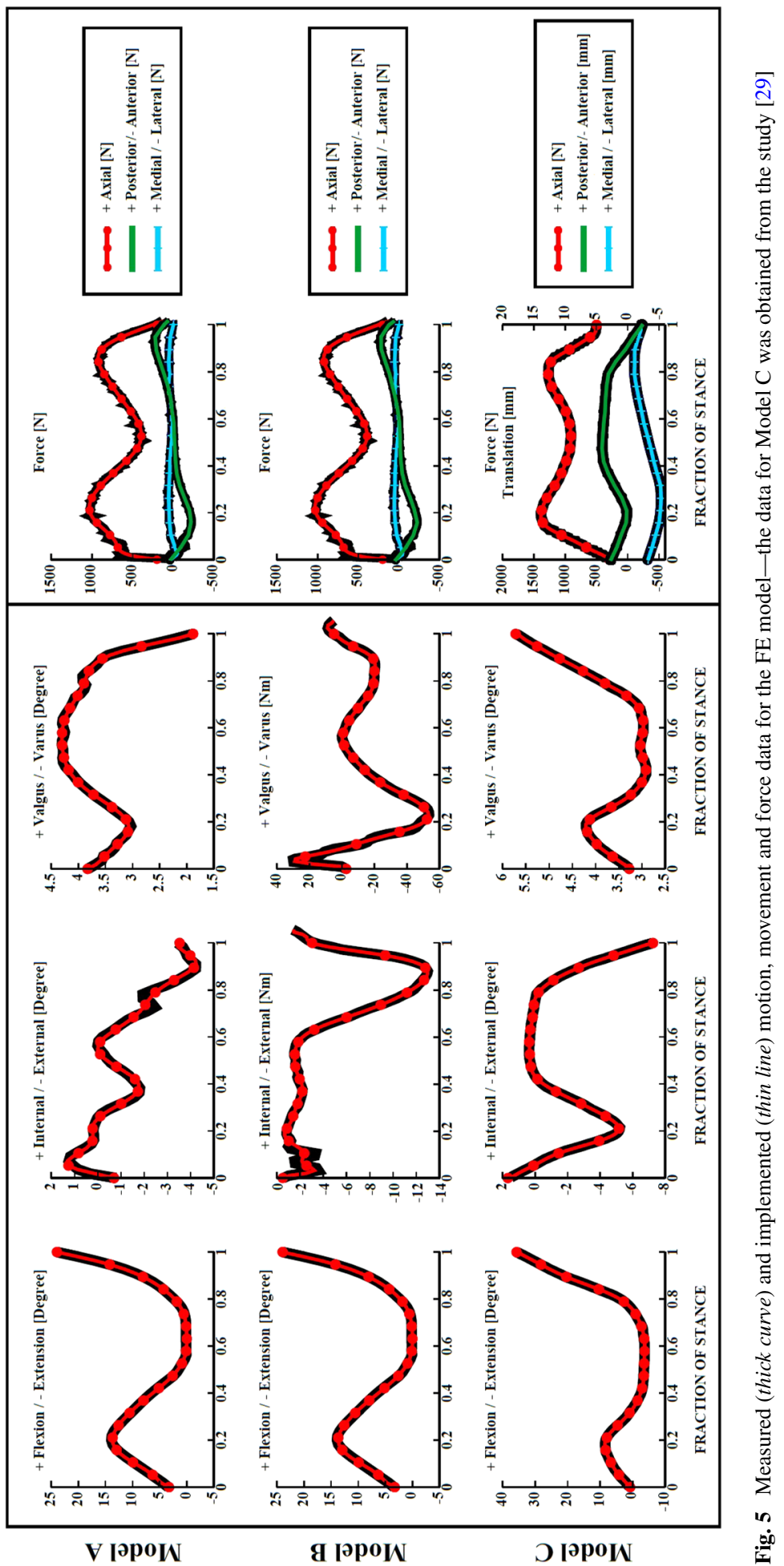




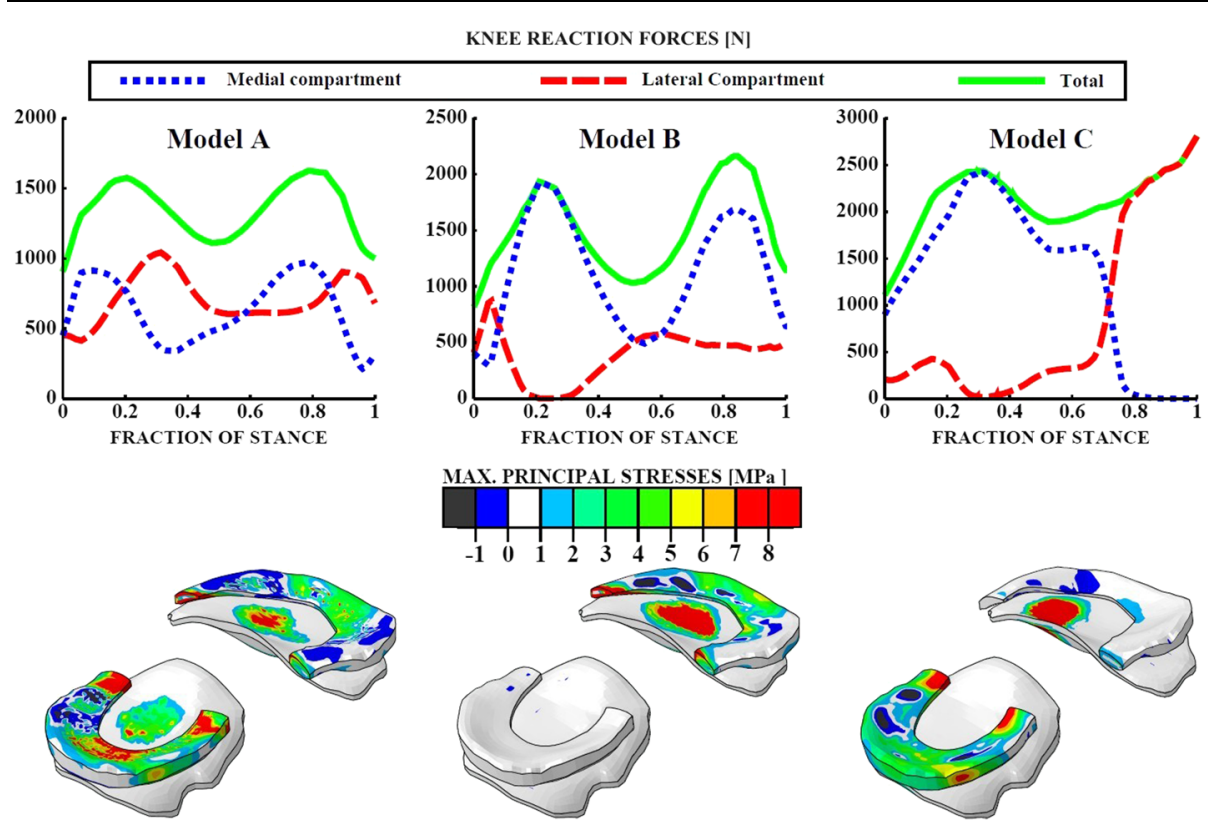

Fig. 6 Reaction forces (top) and maximum principal stress distributions (bottom) in the knee joint in different models - stress distributions are shown at the $20 \%$ of the stance

Fig. 7 Knee rotation angles and translations in different models: the black curve at the top-left indicates the maximal measured varus-valgus rotation produced by the authors of [53], whereas the grey color indicates the range, reaching the varus of $-6^{\circ}$ at the end of stance (not seen in figure)
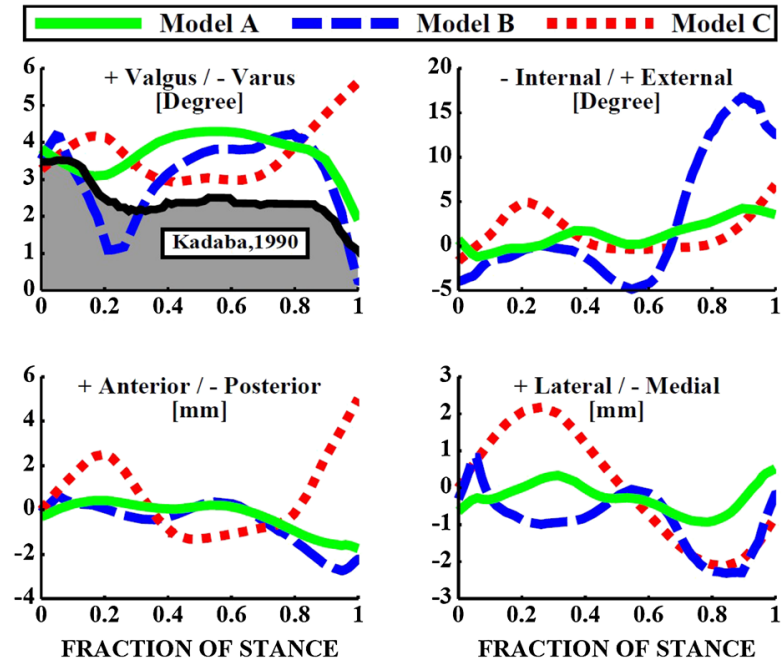

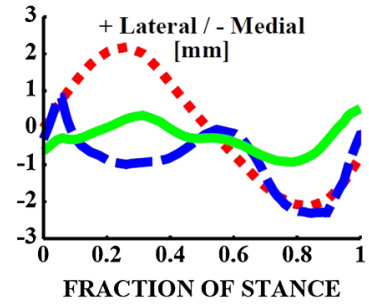

first $60 \%$ of the stance. During 75-100\% of the stance the lateral compartment was exposed to $85-100 \%$ of the total reaction force (Fig. 6, right).

Models A and B showed similar trends in varus-valgus angles, as shown experimentally by [53] (Fig. 7, top-left). The varus-valgus rotation in Model C (literature input) behaved inversely. Internal-external rotation angles were the smallest in A and greatest in B, especially at $\sim 80 \%-\sim 100 \%$ of the stance. Anterior-posterior and lateral-medial translations were mostly within $\pm 1 \mathrm{~mm}$ for Models A and B; however, during the last $20 \%$ of the stance, 


\begin{tabular}{|c|c|c|c|}
\hline & Contact pressure [MPa] (medial compartment) & 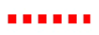 & Contact pressure [MPa] (lateral compartment) \\
\hline$=\square=$ & Max. principal strain [\%] (medial compartment) & 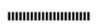 & Max. principal strain [\%] (lateral compartment) \\
\hline
\end{tabular}
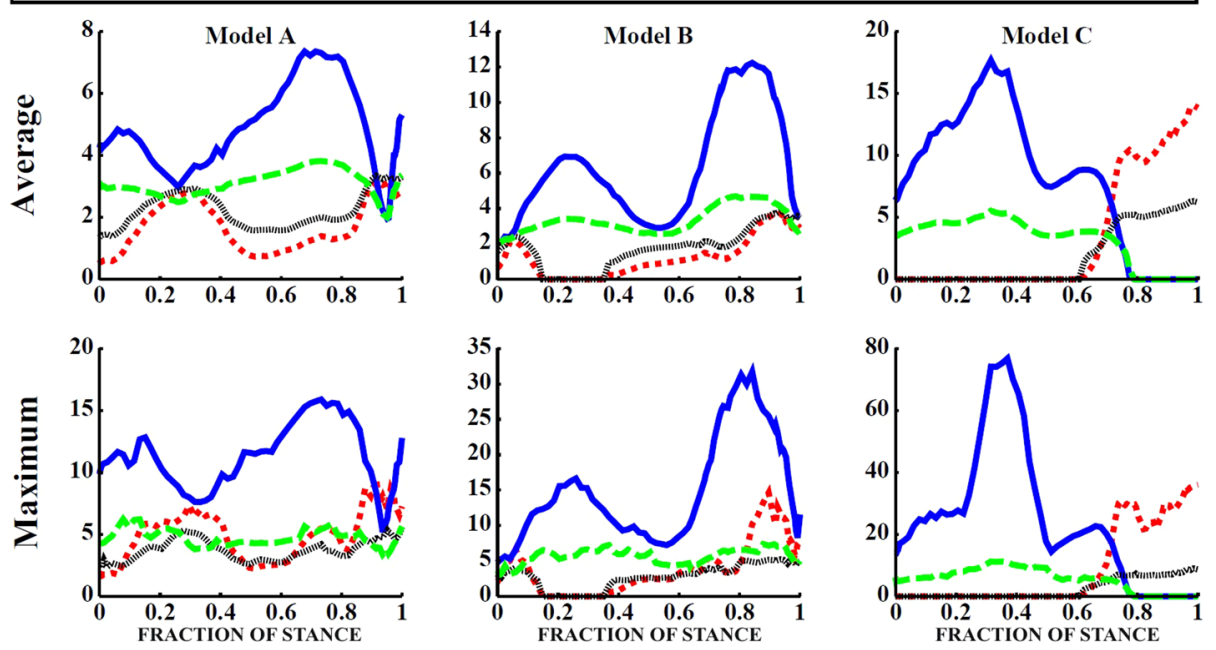

Fig. 8 Average and maximum contact pressures and maximum principal strains in different models-average values calculated over the cartilage-cartilage contact area

a maximum posterior translation of $2 \mathrm{~mm}$ was observed in Models A (Fig. 7, bottom row). Model C showed substantially larger translations than the subject-specific Models A and B.

The contact pressures and maximum principal strains of the tibial cartilage, found at the tibio-femoral contact area, showed similar time-dependent behavior to the reaction forces (Figs. 5 and 6). In Models A and B, the values were lower in the lateral than in the medial compartment, though there was more even distribution of contact pressures and strains in $\mathrm{A}$. In Model C, the contact pressures and maximum principal strains concentrated either on the lateral or medial tibial cartilage, depending on the stance phase (Fig. 8). In Models A and B, the average values of contact pressure and maximum principal strain over the contact area were about half the maximum values (Fig. 8, left \& middle). In Model C, the corresponding average values were three to four times lower than the maximum values (Fig. 8, right).

The depth-wise maximum principal stresses, pore pressures, and fibril strains showed trends similar to the contact pressures (Figs. 8 and 9). The highest average values for the maximum principal stress were located at the interface between the first and the second element layer of the medial tibial cartilage. The lowest values in the medial compartment were found at the cartilage-bone interface. Identical depth-wise stress behaviors were observed in the lateral joint compartment (Fig. 9, left column). The depth-wise fibril strain followed the behavior of the maximum principal stress. The highest fibril strains were found mainly at the superficial zones, either at the surface or at the interface between the element layers 1 and 2 of the medial tibial plateau (Fig. 9, right column). Depending on the stance phase, the lowest values occurred either at the cartilage-bone interface or at the interface between the element layers 3 and 4 . The smallest depth-wise changes were observed in the pore pressure (Fig. 9, middle column). The maximum values were found mainly at the surface layer, while in Model B, the maximum values were found at the cartilage-bone interface (Fig. 9, middle column).

Simulated ACL and PCL elongations were similar in Models A and B, while Model C showed different trends (Fig. 10). The length of the LCL changed similarly in all models, 


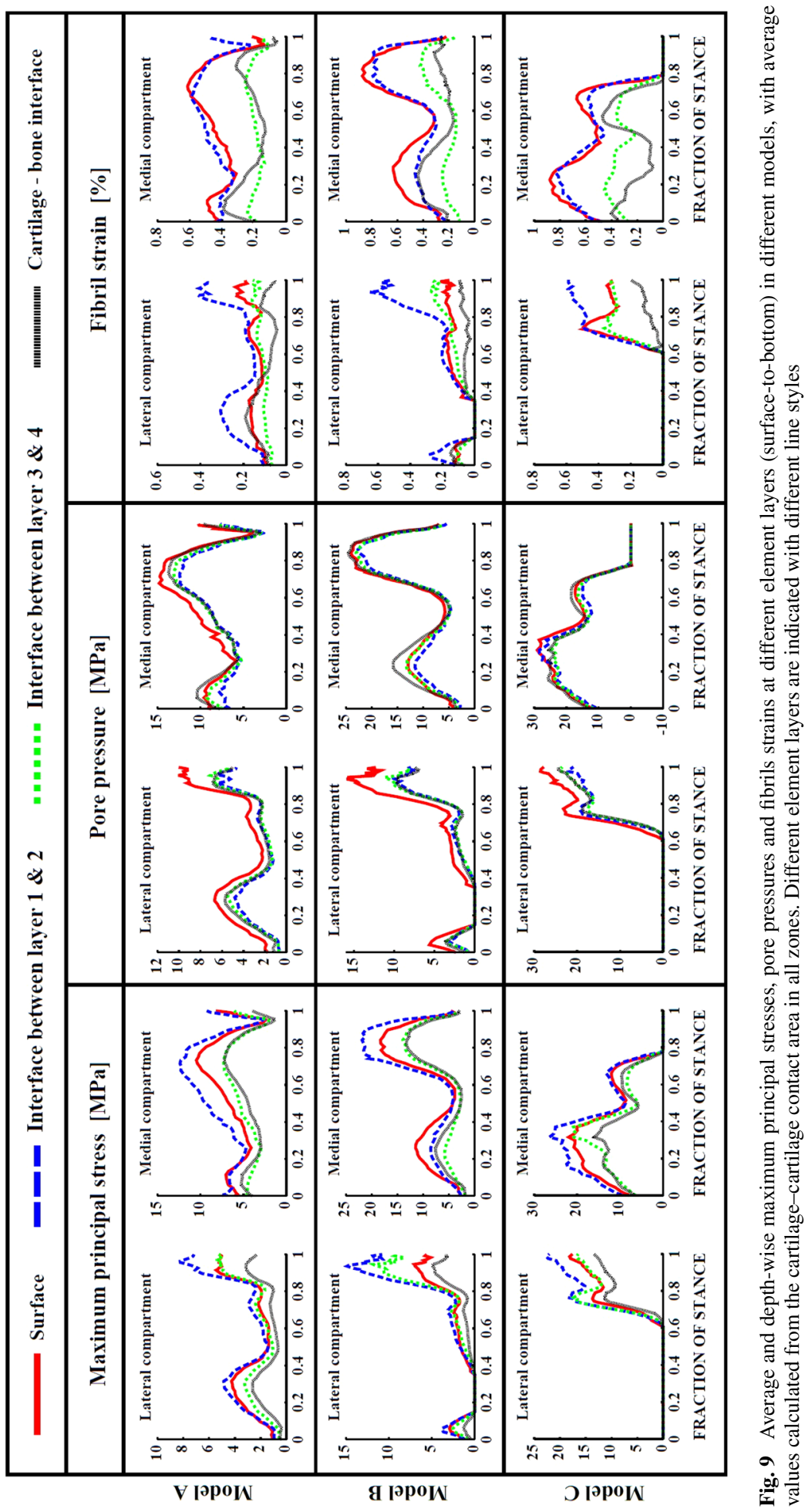


Fig. 10 Elongations of the ACL, PCL, LCL, and MCL simulated

with different models

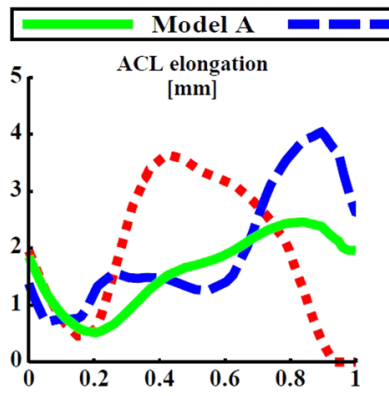

Model B $= \pm \square=$ Model C
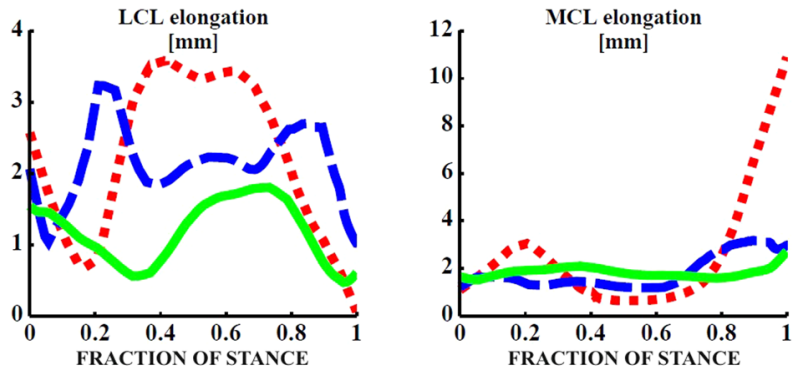

however, the minimum and maximum values were observed at the earliest time points in Model B and the latest time points in Model A. The smallest maximum increase in LCL length was observed in model A $(1.9 \mathrm{~mm})$. The maximum increases in PCL and MCL lengths were substantially higher in Model C (9.5 and $10.9 \mathrm{~mm})$ than in either Models A or B. In Model A, the lengths were 1.6 and $2.7 \mathrm{~mm}$, while in Model B they were 1.8 and $3.2 \mathrm{~mm}$. Between Models A and B, the largest ligament elongation difference $(2.2 \mathrm{~mm})$ was found for the LCL during the first peak loading force of the stance.

\section{Discussion and summary}

For the first time, subject-specific motion analysis, MRI, multibody simulation, and knee joint FE analysis using fibril reinforced material properties of cartilage were combined to simulate the human knee loading that occurs during walking. The model input was based on the rotations (Model A) or moments (Model B) of the knee joint. For comparison, gait data from the literature was the input in the third model (Model C). The knee reaction force, stress and strain trends were similar during the entire stance phase of the gait in Models A and B. However, the distribution of these parameters between the lateral and medial compartments was more even in Model A. Furthermore, the values of depth-wise maximum principal stresses and fibril strains were substantially different between these models. Model $\mathrm{C}$ provided highly uneven stress and strain distributions between the joint compartments.

In Model $\mathrm{C}$, the values for the analyzed parameters were quite different compared to those from the models with subject-specific data. For instance, the maximum principal stresses in the superficial zone of cartilage in Model $\mathrm{C}$ were $\sim 20-26 \mathrm{MPa}$ and the contact pressures were at most $15 \mathrm{MPa}$ or more. This could indicate a risk of cartilage damage $[54,55]$. Since normal walking should not present a risk of cartilage damage, the movement data available from the literature (for different subject) produces unrealistic results. 
The corresponding values for the moment-driven model (Model B) were $\sim 15-20 \mathrm{MPa}$ and $\sim 4-12 \mathrm{MPa}$ for the maximum principal stress and contact pressure, respectively. This range might be more realistic but the values in the medial compartment were still quite high. Patient-specific ligament properties or pre-tensions implemented separately for each ligament could improve the accuracy of Model B. The aforementioned maximum stress and contact pressure values were $\sim 8-12 \mathrm{MPa}$ and $\sim 3-7 \mathrm{MPa}$, respectively, for the rotationdriven model (Model A). The load distribution, as noticed from the forces entering through the joint, between the lateral and medial compartments was also more balanced in this model compared to Models B and C, as also suggested earlier experimentally [56]. Thus, it may estimate loading patterns in the knee cartilage the most realistically. This is supported by earlier studies suggesting that contact stresses in a normal knee should be below $15 \mathrm{MPa}$ [57], though even clearly over $20 \mathrm{MPa}$ stresses for individual joints have been shown [58]. After knee arthroplasty, knee contact stresses have been shown to be over $25 \mathrm{MPa}$ [59]. Even the maximum contact pressures in a single element (average node point values over the element) were less than $15 \mathrm{MPa}$ in Model $\mathrm{A}$, while those were $\sim 30$ and $\sim 80 \mathrm{MPa}$ in Models B and C, respectively. Actually, the study by [57] showed using cadavers that at simulated heel-strike, peak contact stresses in the medial and lateral cartilage were $\sim 14$ and $\sim 5.5 \mathrm{MPa}$, respectively, while those at the toe-off were $\sim 14$ and $\sim 6.5 \mathrm{MPa}$. Our corresponding peak values in Model A were extremely consistent with [57]; 12 and $\sim 4 \mathrm{MPa}$ at heel-strike and $\sim 12$ and $\sim 6 \mathrm{MPa}$ at toe-off.

Substantially different stress and force distribution produced by Model C compared to those obtained from Models A and B can be mainly explained by the different varus-valgus rotation. This varus-valgus rotation in Model C (not subject-specific) was several degrees different than that in Model A (subject-specific) (up to $\sim 4$ degrees), and caused obviously substantial variations in force distributions between medial and lateral compartments. Similar behavior was proven in a recent study [60]. To emphasize the sensitivity of the model response to the input data, we observed that $\sim 4$ and $\sim 6$ degree difference in varus-valgus and internal-external rotation, respectively, in Model $\mathrm{C}$ compared to Model A resulted in a free space of several millimeters between contacting joint surfaces in the medial joint compartment at the end of the gait. This indicates non-physiological contact behavior within the knee joint in Model C [56, 61, 62].

Earlier experimental data [53] showed a varus-valgus trend similar to that simulated here for Model A. The varus-valgus angle strongly contributed to the force distribution between the lateral and medial compartments, in agreement with an earlier study [63]. Similarly, the varus-valgus rotation also influenced ligament strains. A high varus angle resulted in a greater ACL and LCL lengthening, whereas a high valgus angle resulted more in increased PCL and MCL lengths. Based on our previous studies [22, 23], medial-lateral translation also contributes to the stress distribution between the medial and lateral compartments and menisci. Here, in Model A, the reaction force, stress and strain distributions between joint compartments, and LCL elongations consistently followed the medial-lateral translation, while Model C produced opposite varus-valgus rotations to Models A and B, leading to a completely different stress and strain distribution between the compartments. The varusvalgus rotation can vary a great deal among patients, and even small differences in the rotation angle $\left( \pm 3-5^{\circ}\right)$ may produce significant $(\sim 50 \%)$ differences in the medial-lateral reaction forces within the knee [64]. Therefore, optimal, patient-specific motion data, not only varus-valgus rotation but also other rotations and translations, would improve the accuracy of any knee joint model.

Consistent with earlier studies [35, 65], the tangentially oriented collagen fibrils together with the knee joint geometry resulted in the greatest cartilage stresses and fibrils strains 
in the superficial tissue. As found also earlier [35, 65], fluid pressure distribution became quite homogeneous throughout the tissue under the contact area. On the other hand, during the loading response, pore pressures were highest at the cartilage-bone interface in the medial tibial cartilage. Since fluid pressure expanded the tissue horizontally and the vertically oriented collagen fibrils in the deep cartilage bent toward the direction of fluid flow [66], relatively high fibril strains in the deep tissue were also observed.

Ligaments can affect knee joint contact forces [51]. Here, the total joint force was assumed to be the sum of the GRFs and forces caused by ligament strain. With the preelongation of $1.5 \mathrm{~mm}$ and elastic properties we used for the ligaments, the knee joint reaction forces in unloaded and loaded Models A and B were similar to those measured experimentally by Kutzner et al. [51]. Obviously, ligament pre-tension at full extension may be different in different ligaments. As far as we know, such experimental data is not available. However, pre-tension should not affect the relative elongation results produced by the Models A (rotation-controlled) and C (input from the literature), because their motion is controlled by angles. The results of Model B (moment-controlled) are more vulnerable to ligament pre-tension and other properties because the torque required is proportional to the mechanical properties of ligaments. Subject-specific ligament properties could improve the models, especially Model B.

The use of linear elastic properties for ligaments may be criticized as a limitation of the current study. In an experimental in vitro study [67], the nonlinearity of ligaments at small strains was shown. However, the ACL responds linearly after a strain of about 2-3\%, especially with a high strain rate, such as $40 \% / s$ [67]. In our models, the strain rate was over $100 \% / \mathrm{s}$ and ACL strain was constantly between $1 \%$ and $6 \%$. Moreover, strain value ranges were 1-6\%, 2-4 \% and 1-3\% in the PCL, MCL and LCL, respectively. Therefore, we think it was feasible to use linear elastic properties for ligaments, especially when modeling walking with a high strain rate.

Some other limitations of the study should also be addressed. Multibody simulation was used here in order to eliminate or minimize the effect of skin movement which occurred during the experimental motion analysis. Since the multibody simulation was based on rigid bodies, we were not able to analyze joint contact forces realistically. This would necessitate inclusion of deformable bodies, inclusion of ligaments in the multibody knee model, and changes to muscle model would also be a necessity. For this reason, we have approximated joint forces to result from the GRF and forces caused by the ligaments (due to ligament stretching). We believe this is an acceptable method because our joint forces were consistent with the literature [51], as also explained earlier. In the future, one of the goals should be to obtain patient-specific force data from the joint surfaces as well.

Determination of boundary conditions for flexion-extension and varus-valgus relation in the multibody simulation could be considered as a limitation. In the intermediate FE model, flexion-extension and varus-valgus relation was studied with flexion angles of 0-90 degrees, while internal-external rotation was kept fixed and varus-valgus was allowed to rotate freely. This produced even $(\sim 50 \%-50 \%$, on average during the simulation) force distribution between medial and lateral compartments, as suggested in the literature during gait $[56,61,62]$. In reality, internal-external rotation would influence the flexion-extension and varus-valgus relationship. However, it should be noted that the produced intermediate model results (varus-valgus rotation) were used as a boundary condition in the multibody model with a small variation ( \pm 2 degrees).

Our FE model did not include the patella and tendons (patella and quadriceps tendons). These limitations may affect the validity of the moment-controlled model (Model B). In order to simulate the effect of altered loading (e.g., as a result of weight loss, ligament 
rupture) or surgical operations (e.g., partial meniscectomy) on knee joint stresses and strains as realistically as possible, a moment/force-controlled model with realistically implemented ligaments, patella, tendons, and muscle forces should be developed. Furthermore, pre-strain of ligaments was accomplished by increasing their measured lengths from the MRI [68]. However, the increase in ligament lengths was less than $5 \%$; thus, it should not affect the results significantly.

In this study, subject-specific rotation and moment-controlled knee joint models were developed and compared to a reference model with joint motion taken from the literature. The rotation-controlled model showed the most even load distribution between the compartments. The simulated stress and strain values were in the range below typical limits of cartilage failure. Other models predicted a more uneven load distribution between the compartments. Furthermore, the maximum principal stress values in the model with the literature input were clearly beyond cartilage failure limits. The results suggest that patient-specific motion data should be implemented for knee joint models. Even though the presented concept with the combination of motion analysis, multibody dynamics and FE analysis still needs improvements in order to develop and introduce a knee joint model with patientspecific muscle inputs, the current version of the model can already be applied for the analysis of sports training methods, sports equipment design, preventive training regimens, post-op recovery procedures, or in OA research in order to optimize or minimize knee joint forces, with the ultimate goal being the prevention of the initiation and progression of OA.

Acknowledgements We acknowledge the Academy of Finland (project \#138574), the National Graduate School of Engineering Mechanics, Finland and the European Research Council under the European Union's Seventh Framework Program (FP/2007-2013)/ERC Grant Agreement no. 281180 for their financial support. We are grateful to the Finnish IT Center for Science (CSC) for technical support and supercomputer time.

\section{References}

1. Dieppe, P.A., Lohmander, L.S.: Pathogenesis and management of pain in osteoarthritis. Lancet 365, 965-973 (2005)

2. Leskinen, J., Eskelinen, A., Huhtala, H., Paavolainen, P., Remes, V.: The incidence of knee arthroplasty for primary osteoarthritis grows rapidly among baby boomers: a population-based study in Finland. Arthritis Rheumatol. 64, 423-428 (2012)

3. Kujala, U.M., Kettunen, J., Paananen, H., Aalto, T., Battié, M., Impivaara, O., Videman, T., Sarna, S.: Knee osteoarthritis in former runners, soccer players, weight lifters, and shooters. Arthritis Rheum. 38, 539-546 (1995)

4. Miyazaki, T., Wada, M., Kawahara, H., Sato, M., Baba, H., Shimada, S.: Dynamic load at baseline can predict radiographic disease progression in medial compartment knee osteoarthritis. Ann. Rheum. Dis. 61(7), 617-622 (2002)

5. Roos, H., Adalberth, T., Dahlberg, L., Lohmander, L.S.: Osteoarthritis of the knee after injury to the anterior cruciate ligament or meniscus: the influence of time and age. Osteoarthr. Cartil. 3, 261-267 (1995)

6. Brand, K.D., Radin, E.L., Dieppe, P.A., van de Putte, L.: Yet more evidence that osteoarthitis is not cartilage disease. Ann. Rheum. Dis. 65, 1261-1264 (2006)

7. Arokoski, J.P., Jurvelin, J.S., Väätäinen, U., Helminen, H.J.: Normal and pathological adaptations of articular cartilage to joint loading. Scand. J. Med. Sci. Sports 10, 186-198 (2000)

8. Bisseling, R.W., Hof, A.L.: Handling of impact forces in inverse dynamics. J. Biomech. 39, 2438-2444 (2006)

9. Blajer, W., Dziewiecki, K., Mazur, Z.: Multibody modeling of human body for the inverse dynamics analysis of sagittal plane movements. Multibody Syst. Dyn. 18, 217-232 (2007)

10. Kłodowski, A., Rantalainen, T., Mikkola, A., Heinonen, A., Sievänen, H.: Flexible multibody approach in forward dynamic simulation of locomotive strains in human skeleton with flexible lower body bones. Multibody Syst. Dyn. 25(4), 395-409 (2011) 
11. Neptune, R.R., Kautz, S.A., Zajac, F.E.: Contributions of the individual ankle plantar flexors to support, forward progression and swing initiation during walking. J. Biomech. 34(11), 1387-1398 (2001)

12. Alonso, J., Romero, F., Pàmies-Vilà, R., Lugrís, U., Font-Llagunes, J.M.: A simple approach to estimate muscle forces and orthosis actuation in powered assisted walking of spinal cord-injured subjects. Multibody Syst. Dyn. 28, 109-124 (2012)

13. Kłodowski, A., Valkeapää, A., Mikkola, A.: Pilot study on proximal femur strains during locomotion and fall-down scenario. Multibody Syst. Dyn. 28(3), 239-256 (2012)

14. Al Nazer, R., Klodowski, A., Rantalainen, T., Heinonen, A., Sievänen, H., Mikkola, A.: A full body musculoskeletal model based on flexible multibody simulation approach utilized in bone strain analysis during human locomotion. Comput. Method Biomech. Biomed. Eng. 14(6), 573-579 (2011)

15. Al Nazer, R., Klodowski, A., Rantalainen, T., Heinonen, A., Sievänen, H., Mikkola, A.: Analysis of dynamic strains in tibia during human locomotion based on flexible multibody approach integrated with magnetic resonance imaging technique. Multibody Syst. Dyn. 20(4), 287-306 (2008)

16. Geogi, T.: Clinical significance of bone changes in osteoarthritis. Ther. Adv. Musculoskelet. Dis. 4, 259267 (2012)

17. DiSilvestro, M.R., Suh, J.-K.F.: A cross-validation of the biphasic poroviscoelastic model of articular cartilage in unconfined compression, indentation, and confined compression. J. Biomech. 34, 519-525 (2001)

18. Julkunen, P., Kiviranta, P., Wilson, W., Jurvelin, J.S., Korhonen, R.K.: Characterization of articular cartilage by combining microscopic analysis with a fibril-reinforced finite-element model. J. Biomech. 40, 1862-1870 (2007)

19. Adouni, M., Shirazi-Adl, A., Shirazi, R.: Computational biodynamics of human knee joint in gait: from muscle forces to cartilage stresses. J. Biomech. 45, 2149-2156 (2012)

20. Dabiri, Y., Li, L.P.: Altered knee joint mechanics in simple compression associated with early cartilage degeneration. Comput. Math. Methods Med. 2013, 862903 (2013)

21. Kazemi, M., Li, L.P., Buschmann, M.D., Savard, P.: Partial meniscectomy changes fluid pressurization in articular cartilage in human knees. J. Biomech. Eng. 134, 021001 (2012)

22. Mononen, M.E., Jurvelin, J.S., Korhonen, R.K.: Effects of radial tears and partial meniscectomy of lateral meniscus on the knee joint mechanics during the stance phase of the gait cycle-a 3D finite element study. J. Orthop. Res. Off. Publ. Orthop. Res. Soc. 31(8), 1208-1217 (2013)

23. Mononen, M.E., Jurvelin, J.S., Korhonen, R.K.: Implementation of a gait cycle loading into healthy and meniscectomised knee joint models with fibril-reinforced articular cartilage. Comput. Methods Biomech. Biomed. Eng. 18(2), 141-152 (2013)

24. Netravali, N.A., Koo, S., Giori, N.J., Andriacchi, T.P.: The effect of kinematic and kinetic changes on meniscal strains during gait. J. Biomech. Eng. 133, 011006 (2011)

25. Saveh, A.H., Katouzian, H.R., Chizari, M.: Measurement of an intact knee kinematics using gait and fluoroscopic analysis. Knee Surg. Sports Traumatol. Arthrosc. 19, 267-272 (2011)

26. Yang, N.H., Nayeb-Hashemi, H., Canavan, P.K., Vaziri, A.: Effect of frontal plane tibiofemoral angle on the stress and strain at the knee cartilage during the stance phase of gait. J. Orthop. Res. Off. Publ. Orthop. Res. Soc. 28, 1539-1547 (2010)

27. Julkunen, P., Wilson, W., Isaksson, H., Jurvelin, J., Herzog, W., Korhonen, R.: A review of the combination of experimental measurements and fibril-reinforced modeling for investigation of articular cartilage and chondrocyte response to loading. Comput. Math. Methods Med. 2013, 23 (2013)

28. Wilson, W., van Donkelaar, C.C., van Rietbergen, B., Ito, K., Huiskes, R.: Erratum to 'Stresses in the local collagen network of articular cartilage: a poroviscoelastic fibril-reinforced finite element study' [J. Biomech. 37, 357-366 (2004)] and 'A fibril-reinforced poroviscoelastic swelling model for articular cartilage’ [J. Biomech. 38, 1195-1204 (2005)]. J. Biomech. 38, 2138-2140 (2005)

29. Kozanek, M., Hosseini, A., Liu, F., Van de Velde, S.K., Gill, T.J., Rubash, H.E., Li, G.: Tibiofemoral kinematics and condylar motion during the stance phase of gait. J. Biomech. 42, 1877-1884 (2009)

30. Baughman, L.D.: Development of an interactive computer program to produce body description data, Dayton (1983)

31. Engin, A.E., Chen, S.-M.: Human joint articulation and motion-resistive properties, Ohio (1987)

32. Eycleshymer, A.C., Shoemaker, D.M.: A Cross-Section Anatomy. McGraw-Hill, New Yourk (1970)

33. Kłodowski, A.: Flexible Multibody Approach in Bone Strain Estimation During Physical Activity. Lappeenranta University of Technology, Lappeenranta (2012)

34. Momersteeg, T.J., Blankevoort, L., Huiskes, R., Kooloos, J.G., Kauer, J.M., Hendriks, J.C.: The effect of variable relative insertion orientation of human knee bone-ligament-bone complexes on the tensile stiffness. J. Biomech. 28, 745-752 (1995)

35. Mononen, M.E., Julkunen, P., Toyras, J., Jurvelin, J.S., Kiviranta, I., Korhonen, R.K.: Alterations in structure and properties of collagen network of osteoarthritic and repaired cartilage modify knee joint stresses. Biomech. Model. Mechanobiol. 10, 357-369 (2011) 
36. Wilson, W., Van Donkelaar, C.C., Van Rietbergen, B., Ito, K., Huiskes, R.: Stresses in the local collagen network of articular cartilage: a poroviscoelastic fibril-reinforced finite element study. J. Biomech. 37(3), 357-366 (2004)

37. Korhonen, R.K., Laasanen, M.S., Töyräs, J., Rieppo, J., Hirvonen, J., Helminen, H.J., Jurvelin, J.S.: Comparison of the equilibrium response of articular cartilage in unconfined compression, confined compression and indentation. J. Biomech. 35, 903-909 (2002)

38. Korhonen, R.K., Laasanen, M.S., Töyräs, J., Lappalainen, R., Helminen, H.J., Jurvelin, J.S.: Fibril reinforced poroelastic model predicts specifically mechanical behavior of normal, proteoglycan depleted and collagen degraded articular cartilage. J. Biomech. 36, 1373-1379 (2003)

39. Armstrong, C.G., Mow, V.C.: Variations in the intrinsic mechanical properties of human articular cartilage with age, degeneration, and water content. J. Bone Jt. Surg., Am. Vol. 64, 88-94 (1982)

40. Shapiro, E.M., Borthakur, A., Kaufman, J.H., Leigh, J.S., Reddy, R.: Water distribution patterns inside bovine articular cartilage as visualized by $1 \mathrm{H}$ magnetic resonance imaging. Osteoarthr. Cartil. 9(6), 533538 (2001)

41. Mononen, M.E., Mikkola, M.T., Julkunen, P., Ojala, R., Nieminen, M.T., Jurvelin, J.S., Korhonen, R.K.: Effect of superficial collagen patterns and fibrillation of femoral articular cartilage on knee joint mechanics—a 3D finite element analysis. J. Biomech. 45(3), 579-587 (2012)

42. Below, S., Arnoczky, S.P., Dodds, J., Kooima, C., Walter, N.: The split-line pattern of the distal femur: a consideration in the orientation of autologous cartilage grafts. Arthroscopy 18, 613-617 (2002)

43. Benninghoff, A.: Form und Bau der Gelenkknorpel in ihren Beziehungen zur Function. II. Der Aufbau des Gelenkknorpels in seinen Beziehungen zur Funktion. Z. Zellforsch. Mikrosk. Anat. 2, 783-862 (1925)

44. Bottcher, P., Zeissler, M., Maierl, J., Grevel, V., Oechtering, G.: Mapping of split-line pattern and cartilage thickness of selected donor and recipient sites for autologous osteochondral transplantation in the canine stifle joint. Vet. Surg. 38, 696-704 (2009)

45. Goodwin, D.W., Wadghiri, Y.Z., Zhu, H., Vinton, C.J., Smith, E.D., Dunn, J.F.: Macroscopic structure of articular cartilage of the tibial plateau: influence of a characteristic matrix architecture on MRI appearance. Am. J. Roentgenol. 182, 311-318 (2004)

46. Leo, B.M., Turner, M.A., Diduch, D.R.: Split-line pattern and histologic analysis of a human osteochondral plug graft. Arthroscopy 20(Supplement 2), 39-45 (2004)

47. Donahue, T.L., Hull, M.L., Rashid, M.M., Jacobs, C.R.: A finite element model of the human knee joint for the study of tibio-femoral contact. J. Biomech. Eng. 124, 273-280 (2002)

48. Vaziri, A., Nayeb-Hashemi, H., Singh, A., Tafti, B.A.: Influence of meniscectomy and meniscus replacement on the stress distribution in human knee joint. Ann. Biomed. Eng. 36, 1335-1344 (2008)

49. Zielinska, B., Donahue, T.L.: 3D finite element model of meniscectomy: changes in joint contact behavior. J. Biomech. Eng. 128, 115-123 (2006)

50. Villegas, D.F., Maes, J.A., Magee, S.D., Donahue, T.L.: Failure properties and strain distribution analysis of meniscal attachments. J. Biomech. 40, 2655-2662 (2007)

51. Kutzner, I., Heinlein, B., Graichen, F., Bender, A., Rohlmann, A., Halder, A., Beier, A., Bergmann, G.: Loading of the knee joint during activities of daily living measured in vivo in five subjects. J. Biomech. 43, 2164-2173 (2010)

52. Komistek, R.D., Stiehl, J.B., Dennis, D.A., Paxson, R.D., Soutas-Little, R.W.: Mathematical model of the lower extremity joint reaction forces using Kane's method of dynamics. J. Biomech. 31, 185-189 (1998)

53. Kadaba, M.P., Ramakrishnan, H.K., Wootten, M.E.: Measurement of lower extremity kinematics during level walking. J. Orthop. Res. 8, 383-392 (1990)

54. Kempson, G.E., Spivey, C.J., Swanson, S.A.V., Freeman, M.A.R.: Patterns of cartilage stiffness on normal and degenerate human femoral heads. J. Biomech. 4(6), 597-609 (1971)

55. Woo, S.-Y., Akeson, W.H., Jemmott, G.F.: Measurements of nonhomogeneous, directional mechanical properties of articular cartilage in tension. J. Biomech. 9, 785-791 (1976)

56. Zhao, D., Banks, S.A., Mitchell, K.H., D’Lima, D.D., Colwell, C.W., Fregly, B.J.: Correlation between the knee adduction torque and medial contact force for a variety of gait patterns. J. Orthop. Res. 25(6), 789-797 (2007)

57. Thambyah, A., Goh, J.C., De, S.D.: Contact stresses in the knee joint in deep flexion. Med. Eng. Phys. 27(4), 329-335 (2005)

58. Brand, R.A.: Joint contact stress: a reasonable surrogate for biological processes? Iowa Orthop. J. 25, 82 (2005)

59. D’Lima, D.D., Steklov, N., Fregly, B.J., Banks, S.A., Colwell, C.W.: In vivo contact stresses during activities of daily living after knee arthroplasty. J. Orthop. Res. 26(12), 1549-1555 (2008)

60. Adouni, M., Shirazi-Adl, A.: Evaluation of knee joint muscle forces and tissue stresses-strains during gait in severe OA versus normal subjects. J. Orthop. Res. 32(1), 69-78 (2014) 
61. Zhao, D., Banks, S.A., D’Lima, D.D., Colwell, C.W., Fregly, B.J.: In vivo medial and lateral tibial loads during dynamic and high flexion activities. J. Orthop. Res. 25(5), 593-602 (2007)

62. Mononen, M.E., Jurvelin, J.S., Korhonen, R.K.: Implementation of a gait cycle loading into healthy and meniscectomised knee joint models with fibril-reinforced articular cartilage. Comput. Methods Biomech. Biomed. Eng. 18(2), 141-152 (2015)

63. Guess, T.M., Thiagarajan, G., Kia, M., Mishra, M.: A subject specific multibody model of the knee with menisci. Med. Eng. Phys. 32, 505-515 (2010)

64. D’Lima, D.D., Fregly, B.J., Patil, S., Steklov, N., Colwell, C.W.: Knee joint forces: prediction, measurement, and significance. Proc. Inst. Mech. Eng., H J. Eng. Med. 226, 95-102 (2012)

65. Räsänen, L.P., Mononen, M., Nieminen, M.T., Lammentausta, E., Jurvelin, J.S., Korhonen, R.K.: Implementation of subject-specific collagen architecture of cartilage into a $2 \mathrm{D}$ computational model of a knee joint-data from the osteoarthritis initiative (OAI). J. Orthop. Res. 31, 10-22 (2013)

66. Shirazi, R., Shirazi-Adl, A., Hurtig, M.: Role of cartilage collagen fibrils networks in knee joint biomechanics under compression. J. Biomech. 41, 3340-3348 (2008)

67. Pioletti, D.P., Rakotomanana, L.R., Leyvraz, P.-F.: Strain rate effect on the mechanical behavior of the anterior cruciate ligament-bone complex. Med. Eng. Phys. 21, 95-100 (1999)

68. Gantoi, F.M., Brown, M.A., Shabana, A.A.: Finite element modeling of the contact geometry and deformation in biomechanics applications. J. Comput. Nonlinear Dyn. 8(4), 041013 (2013) 